\title{
On the Estimation the Probability of Cardiovascular and Cerebrovascular Events in Hypertensive Patients Using Nonlinear Analysis, Time and Frequency Domain Methods ${ }^{+}$
}

\author{
José María López Belinchón ${ }^{1}$, Miguel Ángel López Guerrero ${ }^{2}$ and Raúl Alcaraz Martínez ${ }^{3}$ \\ 1 SIDIS Research Group, Department of Mathematics, Polytechnic School of Cuenca, University of Castilla-La \\ Mancha, Cuenca, Spain \\ 2 SIDIS Research Group, Department of Mathematics and Institute of Applied Mathematics in Science and \\ Engineering (IMACI), Polytechnic School of Cuenca, University of Castilla-La Mancha, Cuenca, Spain \\ 3 Research Group in Electronic, Biomedical and Telecommunication Engineering, University of Castilla-La \\ Mancha (UCLM), Cuenca, Spain \\ † Presented at the Entropy 2021: The Scientific Tool of the 21st Century, 5-7 May 2021; Available online: \\ https://sciforum.net/conference/Entropy2021/.
}

Published: 5 May 2021

Applications of entropy-based parameters to the time series generated by physiological systems of the human body are revealing hidden information of interest, which is allowing to improve diagnosis and treatment of several diseases [1]. In this context, a widely analysed physiological time series is the heart rate variability, obtained from the Surface electrocardiograma (ECG) [2]. For example, its entropybased analysis has allowed to detect abnormalities associated with letal and non-letal cardiac arrhythmias. In addition to provide information about the instantaneous heart rhythm, it is today accepted that this time series reflects the behavior of the autonomic nervous system.

In this study, the heart rate variability is analyse to anticipate the risk of occurence of an adverse cardiovascular event in hypertensive patients (i.e., myocardial infarction, stroke, syncopal event, etc.), which is the pathology with the most prevalence amongst the developed countries [3]. Briefly, several entropy-based measures haven been applied to the heart rate time series derived from $24 \mathrm{~h}$-length ECG signals acquired from 139 patients, who were followed during 1 year. These parameters consist of sample entropies, Fuzzy-based entropies, symbolic entropies, etc.

Entropy measures based on different approaches, such irregularity, symbolization, ordinal pattern quantification, etc. were analyzed these indices were only able to discern between hypertensive and non-hypertensive patients who suffered an event within the follow up time, with a 70\% accuracy, their combination with other common time and frequency domain parameters estimated from heart rate variability improved diagnostic accuracy to about $80 \%$ [4].

As a conclusion, nonlinear analysis of the heart rate variability provided by entropy-based measures provides complementary information to other linear indexes, at high-risk of developing future cardiovascular events, which can lead them to imminent death.

\section{References}

1. Goldberger, A.L. Non-linear dynamics for clinicians: Chaos theory, fractals, and complexity at the bedside. Lancet 1996, 347, 1312-1314.

2. Goldberger, A.L.; Peng, C.K.; Lipsitz, L.A. What is physiologic complexity and how does it change with aging and disease? Neurobiol. Aging 2002, 23, 23-26. 
3. Spasic, S.Z.; Kesic, S. Nonlinearity in living systems: Theoretical and practical perspectives on metrics of physiological signal complexity. Front. Physiol. 2019, 10, 298.

4. Solaro, N.; Malacarne, M.; Pagani, M.; Lucini, D. Cardiac baroreflex, HRV, and statistic: An interdisciplinary approach in hypertension. Front. Physiol. 2019, 10, 478.

(c) (1)

(C) 2021 by the authors. Licensee MDPI, Basel, Switzerland. This article is an open access article distributed under the terms and conditions of the Creative Commons Attribution (CC BY) license (http://creativecommons.org/licenses/by/4.0/). 